\title{
The slow formation of a pitch percept beyond the ending time of a short tone burst
}

\author{
LAURENT DEMANY and CATHERINE SEMAL \\ CNRS and Université Victor Segalen, Bordeaux, France
}

\begin{abstract}
The discriminability of short tone bursts differing in frequency was measured in terms of the sensitivity index $d^{\prime}$ as a function of interstimulus interval (ISI). The two stimuli presented on each trial consisted of either 6 or 30 sinusoidal cycles. When the frequency of the first stimulus varied randomly and widely from trial to trial (Experiment 1), discriminability was maximal for an ISI of about $400 \mathrm{msec}$ in the 6-cycles condition and for a significantly longer ISI (of about $1 \mathrm{sec}$ ) in the 30-cycles condition. However, when the first stimulus had only two possible frequencies and the second stimulus was fixed (Experiment 2), the optimal ISI appeared to be about $400 \mathrm{msec}$ in both conditions. A final experiment confirmed that, for tone bursts of 30 cycles, the optimal ISI was dependent on the perceptual uncertainty of the first stimulus. These results support the idea that the duration required to perceive the pitch of a sound as accurately as possible may far exceed the duration of the stimulus itself. More importantly, they indicate that the required duration is not a constant.
\end{abstract}

Perception takes time, and the amount of time required to perceive one attribute of a given sound as accurately as possible may far exceed the duration of the stimulus itself. Such a view has been supported by a number of auditory discrimination experiments in which performance was assessed as a function of the time interval separating the two stimuli presented on a given trial. When short stimuli were used, it was typically found that discrimination performance improved as the interstimulus interval (ISI) increased, in a range with an upper boundary that was often well above 200 or $300 \mathrm{msec}$. Tanner (1961) may have been the first to report such data. In an experiment on intensity discrimination, he found that performance (measured in terms of $d^{\prime}$ ) was optimal for an ISI of about $600 \mathrm{msec}$, decreased slowly for larger ISIs, and dropped much more abruptly for smaller ISIs. In his brief description of this experiment, Tanner did not specify his stimuli. However, his basic result was replicated by Sorkin (1966), who used 50-msec tone bursts in a similar experiment and also found an optimal ISI of about $600 \mathrm{msec}$ when the two stimuli produced on a given trial were presented to the same ear. Sorkin further observed that the optimal ISI was only slightly smaller if the two stimuli were instead presented to opposite ears. This observation is important because it rules out the idea that the need for an appreciable ISI for optimal performance could be due entirely to a forward masking of the second stimulus by the first one at very short ISIs. Forward masking was minimized when the two stimuli were presented to opposite ears. Thus, at

The authors are grateful to William M. Hartmann for his mathematical advice and to Gaspard Montandon for his technical assistance. Correspondence should be addressed to L. Demany, Laboratoire de Neurophysiologie, BP 63, Université Victor Segalen, 146 rue Léo Saignat, F-33076 Bordeaux, France (e-mail: laurent.demany@psyac.u-bordeaux2.fr). least in this case, the ISI effect clearly meant that several hundreds of milliseconds were needed to perceive the first stimulus optimally after its offset.

Taylor and Smith (1975) and Berliner, Durlach, and Braida (1977) corroborated Tanner's (1961) and Sorkin's (1966) findings in subsequent studies on intensity discrimination. Furthermore, analogous results were obtained for other acoustic continua. For instance, the studies of Small and Campbell (1962) and Carbotte (1973) indicate that it is necessary to use an ISI exceeding $200 \mathrm{msec}$ in order to measure optimal performances in the discrimination of short sounds differing in duration. Several hundreds of milliseconds also seem to be needed for the optimal discrimination of formant transitions in synthetic syllables, according to Pisoni (1973). Finally, in the domain of pitch, an effect of the same type was reported by Massaro and Idson (1977). Their stimuli were 20-msec bursts of sinusoids differing in frequency and, as in Sorkin's study, the two stimuli used on a given trial were presented either to the same ear or to opposite ears. In both cases, discrimination performance, measured in terms of $P(\mathrm{C})$ (percent correct), appeared to improve from a level close to chance for an ISI of $5 \mathrm{msec}$ to an optimum level for an ISI of about $350 \mathrm{msec}$.

Before the study that we just mentioned, Massaro had conducted a series of related experiments in which the listeners' task was not to compare two successive stimuli but instead to make a two-alternative identification of one stimulus, rapidly followed by another stimulus acting as a source of "backward recognition masking" (see, e.g., Massaro, 1970, 1972, 1975). Here also, it was found that performance improved as the ISI increased. To account for the results obtained in these experiments, Massaro (1972; Massaro \& Loftus, 1996) proposed a simple theory. According to this theory, when a brief sound (S1) is presented to a listener, it is initially memorized in a preperceptual 
auditory store (PPAS), where its trace is maintained for a fixed duration independently of the sound itself. The perceptual analysis of the sound corresponds to a processing of the information memorized in this PPAS. If a second sound (S2) is presented very soon after S1, the perceptual analysis of S1 will be prematurely stopped because S2 will replace S1 in the PPAS. Otherwise, the perceptual analysis of S1 will continue until its preperceptual trace disappears. Thus, the time available and needed for an optimal perceptual analysis is the fixed temporal span of the PPAS. In support of the idea that this amount of time is a constant, Massaro and Loftus (1996, p. 75) mentioned an unpublished study in which listeners had to identify three properties of 20-msec test tones followed by a masker: their pitch, loudness, and spatial location. The effect of the ISI on identification performance was found to be the same for all three properties. Massaro and Loftus estimated at about $250 \mathrm{msec}$ the temporal span of the PPAS and the duration required for optimal perceptual analyses. However, this estimate was derived mainly from experiments in which performance was measured in terms of $P(\mathrm{C})$. According to data reported by Massaro himself (see, e.g., Massaro, 1972) as well as by other authors, using $d^{\prime}$ instead might lead to a longer estimate. ${ }^{1}$

In the present study, we again tested the hypothesis that the time required to perceive a short sound as accurately as possible is a constant. Three experiments will be reported. ${ }^{2}$ In all of them, trained listeners had to compare on each trial the frequencies (pitches) of two successive tone bursts separated by a variable silent ISI. These tone bursts were either very short (6 sinusoidal cycles) or still short but five times longer (30 sinusoidal cycles). Moreover, the frequency of the first tone burst was sometimes selected at random from a very wide range, thus making this stimulus highly unpredictable, whereas in other conditions it took only a small number of possible values. Our aim was to determine whether or not the optimal ISI would depend on such factors.

\section{EXPERIMENT 1}

\begin{abstract}
Method
Subjects. Four listeners with no known hearing deficits, including the first author (L.D., in his late 40s), took part as subjects in the experiment. Subjects G.M., M.B., and S.C. were in their 20s. Except for M.B., each subject had previously participated in other experiments concerned with pitch discrimination.

Stimuli and Procedure. The temporal fine structure of the stimuli consisted of exactly 6 sinusoidal cycles in one experimental condition and exactly 30 sinusoidal cycles in the other condition; each sinusoid started and ended at a positive-going zero crossing. The stimuli had an amplitude envelope consisting of 1 cycle of a raised cosine function $[1+\cos (\varphi), \varphi$ running from $-\pi$ to $+\pi \mathrm{rad}]$. Their amplitude spectra were much broader in the 6-cycles condition than in the 30-cycles condition, as is shown by Figure 1. S1, the first stimulus presented within a trial, had a frequency that was selected randomly between 400 and $2400 \mathrm{~Hz}$; the corresponding probability distribution was rectangular on a logarithmic frequency scale. The second stimulus, S2, was equiprobably lower or higher in frequency. The subject's task was to identify the direction of this
\end{abstract}

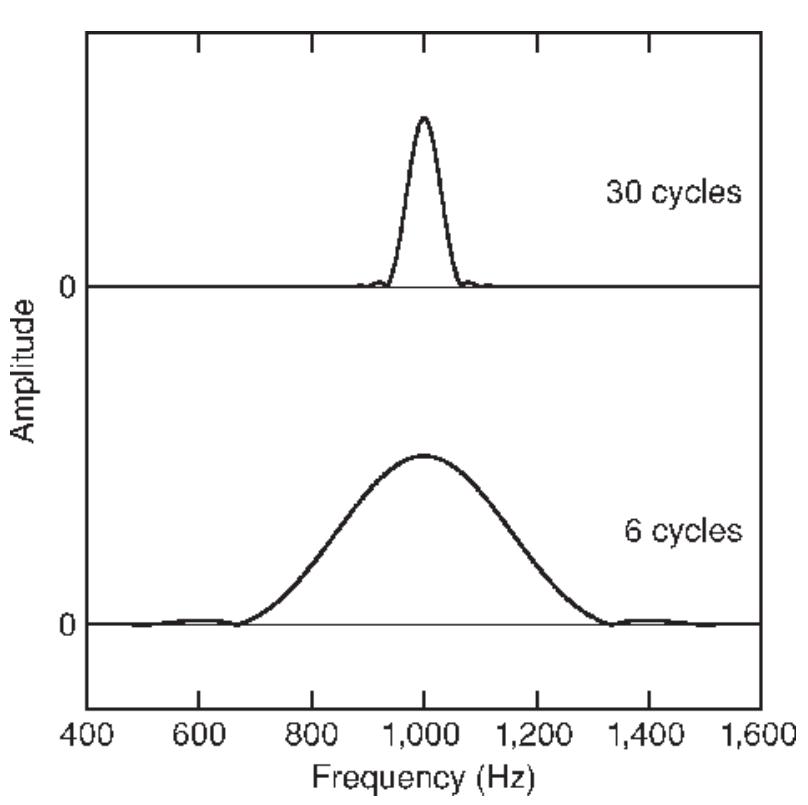

Figure 1. Amplitude spectra of the stimuli produced in the 6cycles and 30-cycles conditions for a nominal frequency of $1000 \mathrm{~Hz}$. Each spectrum consists of a main lobe peaking at $1000 \mathrm{~Hz}$ and a series of lateral lobes decreasing in size with distance from the peak. Only the first lateral lobe on each side is discernible in the figure; its peak is $31.5 \mathrm{~dB}$ below the main peak. Note that the spectrum is much broader in the 6-cycles condition than in the 30-cycles condition.

change. It could be anticipated from previous research (e.g., Moore, 1973) that the perceptual sensitivity to a given frequency change would be much poorer in the 6-cycles condition than in the 30 -cycles condition.

Within each condition, since the number of sine cycles was fixed, the duration of a stimulus was inversely proportional to its frequency. The duration range of S1 was $2.5-15 \mathrm{msec}$ in the 6-cycles condition and $12.5-75 \mathrm{msec}$ in the 30 -cycles condition. Because it was desirable to equate all stimuli in loudness and thus to take into account the effect of duration on loudness (Scharf, 1978), all the stimuli were equated in energy. Therefore, peak amplitude covaried with duration and thus with frequency. For S1, the maximum peak amplitude (obtained in the 6-cycles condition at $2400 \mathrm{~Hz}$ ) corresponded to a sound pressure level of $88 \mathrm{~dB}$. Although the frequency shift from $\mathrm{S} 1$ to $\mathrm{S} 2$ was associated with a change in duration, it was always small enough to affect only pitch from the perceptual point of view. The auditory system's sensitivity to a change in duration is markedly poorer than its sensitivity to a change in frequency. ${ }^{3}$

The stimuli were generated via a 24-bit digital-to-analog converter (Echo Gina) at a sampling rate of $44.1 \mathrm{kHz}$. They were presented binaurally, by means of Sennheiser HD265 headphones, in a double-walled soundproof booth. On each trial, the direction of the frequency shift from S1 to S2 was selected at random and the subject responded by pressing one of two buttons; visual feedback was provided immediately after the response via the computer screen, on which the word JUSTE ("correct") or FAUX ("wrong") appeared. Trials were run in blocks of 75 , during which S1 and S2 contained a constant number of sine cycles and were separated by a constant ISI of 200, 350, 500, 1,000, 2,000, or 4,000 msec. In any block of trials, the delay separating a response from the presentation of $\mathrm{S} 1$ in the next trial was equal to the ISI value $+300 \mathrm{msec}$ (see Cowan, Saults, \& Nugent, 1997). When the ISI exceeded $1 \mathrm{sec}$, a countdown 
was displayed on the computer screen during both the ISI and the intertrial delay. For any ISI, therefore, the subject knew precisely when $\mathrm{S} 1$ and $\mathrm{S} 2$ would occur.

In the experiment proper, the relative magnitude of the frequency shift from S1 to S2 - that is, the size of the shift on a logarithmic frequency scale, $\Delta F_{\text {rel }}$-was fixed within each block of trials, as were the number of sine cycles and the ISI. (In order to minimize the variability of discrimination performance from 400 to $2400 \mathrm{~Hz}$, it was preferable to fix $\Delta F_{\text {rel }}$ rather than the frequency difference in hertz.) Across blocks, only two different values of $\Delta F_{\text {rel }}$ (one for each number of sine cycles) were used for a given subject. Our aim was to obtain, for an ISI of $350 \mathrm{msec}$, a $d^{\prime}$ value of about 2.0 in both the 6 -cycles condition and the 30 -cycles condition ( $d^{\prime}$ being defined as the sensitivity to the difference between the two possible directions of the frequency shifts). Thus, $\Delta F_{\text {rel }}$ had to be larger in the 6-cycles condition than in the 30-cycles condition. The appropriate values of $\Delta F_{\text {rel }}$ were estimated during a preliminary stage of the experiment, in which the ISI was fixed at $350 \mathrm{msec}$. This preliminary stage included at least five sessions, each lasting about $1 \mathrm{~h}$, and thus it also served as a training phase. The two $\Delta F_{\text {rel }}$ values eventually selected for each subject are indicated in Table 1; on average, they differed by a factor of about 4.5 .

The experiment proper consisted of 10 sessions, run on different days. In each session, 12 blocks of trials (1 for each of the six ISI values in each condition) were run. From block to block, the 6-cycles and 30-cycles conditions were used alternately and the ISI varied monotonically, from $200 \mathrm{msec}$ to $4 \mathrm{sec}$ in half of the sessions and from $4 \mathrm{sec}$ to $200 \mathrm{msec}$ in the other half.

\section{Results}

For a given subject, number of sine cycles, and ISI, 750 trials were run (in 10 blocks). In the statistical analysis of the data, these 750 trials were treated as five successive subsets of 150 trials, from which five $d^{\prime}$ values were computed. The corresponding means and standard deviations of $d^{\prime}$ are displayed in Figure 2. The four upper panels show the individual results, which are pooled in the lower panel.

It can be seen that the 4 subjects behaved similarly. Moreover, as we expected, $d^{\prime}$ was approximately equal to 2.0 in both the 6-cycles and 30-cycles conditions when the ISI was $350 \mathrm{msec}$. For each number of cycles, as the ISI increased, $d^{\prime}$ first increased and then decreased. The main finding, however, is that the optimal ISI was definitely longer in the 30-cycles condition than in the 6-cycles condition. For each subject, when the ISI increased from 350 or $500 \mathrm{msec}$ to $1 \mathrm{sec}, d^{\prime}$ decreased in the 6-cycles condition but increased in the 30 -cycles condition. An ANOVA

Table 1

$\Delta F_{\text {rel }}$ Values (Expressed in Musical Cents) Used in the 6-Cycles and 30-Cycles Conditions of Experiments 1 and 2

\begin{tabular}{lccccc}
\hline & \multicolumn{2}{c}{ Experiment 1} & & \multicolumn{2}{c}{ Experiment 2} \\
\cline { 2 - 3 } \cline { 5 - 6 } Subject & 6 Cycles & 30 Cycles & & 6 Cycles & 30 Cycles \\
\hline G.M. & 100 & 20 & & 50 & 8 \\
L.D. & 93 & 28 & & 50 & 13 \\
M.B. & 110 & 25 & & & \\
S.C. & 85 & 15 & & 56 & 10 \\
E.D. & & & & 39 & 7 \\
N.P. & & & & 39.8 & 9.5 \\
$\quad$ Mean & 97.0 & 22.0 & & 48.8
\end{tabular}

Note -1 cent $=1 / 100$ semitone $=1 / 1,200$ octave. confirmed the existence of a global interaction between ISI and number of cycles $\left[F(5,20)=26.9, p<10^{-5}\right]$. On average, $d^{\prime}$ was markedly higher in the 30 -cycles condition than in the 6-cycles condition, but of course this effect is not meaningful in itself, since $\Delta F_{\text {rel }}$ did not have the same value in the two conditions.

In order to assess the effect of practice on subjects' performance, we compared the mean $d^{\prime}$ values obtained in the first four experimental sessions with those obtained in the last four (Sessions 7-10). The corresponding data are displayed in Figure 3. Overall, $d^{\prime}$ was about 20\% higher in the last four sessions than in the first four. However, for each number of cycles there was essentially no change in the dependence of $d^{\prime}$ on the ISI.

Given that the frequency and duration of the stimuli varied in a wide range from trial to trial, it was also interesting to assess their effect on performance for each number of cycles. We did so by dissociating the trials on which the frequency of S1 had been higher than $980 \mathrm{~Hz}$ (the geometric mean of 400 and $2400 \mathrm{~Hz}$ ) from those on which it had been lower than $980 \mathrm{~Hz}$. The average $d^{\prime}$ values obtained for the two corresponding classes of stimuli are presented in Figure 4. Overall, $d^{\prime}$ was higher when the frequency of S1 was relatively low and its duration relatively long. However, for each number of cycles, the pattern of the results obtained was essentially independent of frequency and duration. The optimal ISI did not appear to be significantly different above and below $980 \mathrm{~Hz}$. It is important to note, in addition, that although $d^{\prime}$ had the same maximum value (2.3) when $\mathrm{S} 1$ had 6 cycles but a low frequency and when it had 30 cycles but a high frequency, this maximum value of $d^{\prime}$ was nonetheless obtained for a shorter ISI in the former case than in the latter case.

\section{Discussion}

Why did the optimal ISI differ in the 6-cycles and 30 -cycles conditions? On the basis of previous research (Ronken, 1972; Turner, Zeng, Relkin, \& Horwitz, 1992), one can rule out the idea that in either condition the initial rise of $d^{\prime}$ when the ISI increased would stem from a forward masking of S2 by S1 at the shortest ISI or ISIs. Turner et al. found that the frequency difference limen of two 25 -msec tones was not elevated by the presentation, $100 \mathrm{msec}$ before each of them, of a narrowband noise in the same frequency region, even if this noise was much more intense than the tones. Using instead 10-msec tones, Ronken found that if each of these stimuli was immediately preceded by another tone of the same intensity but halfway between them in frequency, this forward "masker" actually improved the frequency difference limen. Hence, it seems clear that in both of our conditions the initial rise of $d^{\prime}$ stemmed from a deleterious effect of S2 on the perception of S1 rather than from a deleterious effect of S1 on the perception of S2. Presumably, as Massaro and Idson (1977) suggested in their related study, optimal perceptual processing of S1 was not possible when S2 occurred soon after it. But why did the perceptual processing of S1 require a longer duration in the 30 -cycles condition than in the 6-cycles condition? The data displayed in Figure 4 

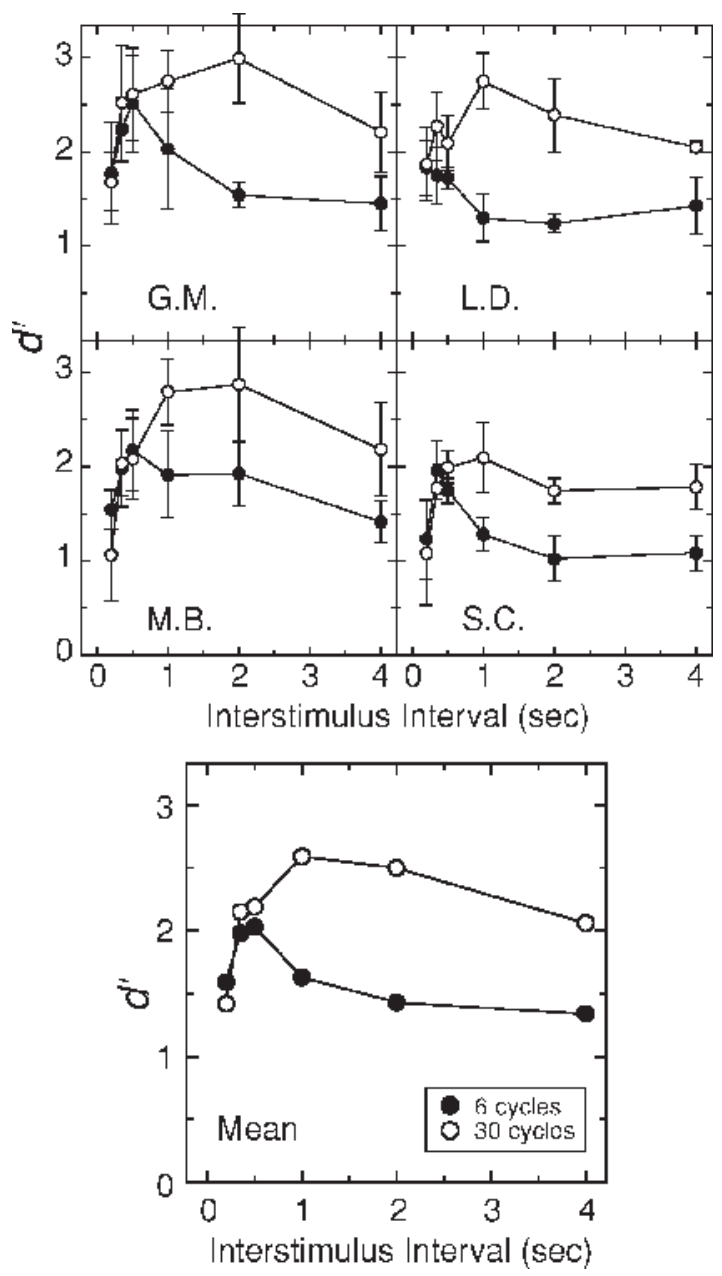

Figure 2. Results of Experiment 1: $d^{\prime}$ as a function of interstimulus interval in the 6-cycles condition (filled circles) and the 30cycles condition (open circles). The four upper panels display the individual data, which are averaged in the bottom panel. $d^{\prime}$ represents the relative discriminability of ascending and descending frequency shifts $(\mathrm{S} 2>\mathrm{S} 1 \mathrm{vs.} \mathbf{S 2}<\mathrm{S1}$ ). The error bars have a total length of two standard deviations.

indicate that this effect is unrelated to the fact that, for the optimal ISI, $d^{\prime}$ was on average higher in the 30 -cycles condition. A consideration of the spectral properties of the stimuli in the two conditions (cf. Figure 1) does not provide an obvious explanation of the effect in question. One possible hypothesis is that, in the 30 -cycles condition, the duration required for optimal perceptual processing was longer because the stimuli carried more information in the temporal domain. If each cycle of a tone provides a temporal cue about its period and the corresponding cues are statistically combined in the pitch measurement process (Goldstein \& Srulovicz, 1977), then the amount of processing required for an optimal pitch measurement will depend on the number of cycles per se. This hypothesis makes sense insofar as there is evidence that the pitch of short tone bursts such as those used here is extracted by the auditory system from temporal rather than spectral (tonotopic) information (Moore, 1973). However, our crucial assumption here is that, for short stimuli, the temporal information provided by sequences of action potentials at a peripheral level of the auditory system can be analyzed "off-line" (i.e., after the stimulus presentation). This assumption is far from trivial.

An alternative hypothesis must be considered. The frequency of S1 varied between the same limits - 400 and $2400 \mathrm{~Hz}$ - in the two conditions, but between these limits there was a much larger number of frequency difference limens in the 30-cycles condition than in the 6-cycles condition. Thus, it is reasonable to assume that from the perceptual point of view, S1 was more variable and less predictable in the 30 -cycles condition. This might be the reason for which the amount of time required to process S1 optimally was larger in the 30 -cycles condition.

A simple way to assess the relative merits of the two hypotheses just mentioned is to replicate Experiment 1 without its roving procedure, in order to minimize the variability of $\mathrm{S} 1$ in each condition. This is what we did in Experiment 2.

\section{EXPERIMENT 2}

\section{Method}

Methodologically, Experiment 2 differed from Experiment 1 in only two respects. First, S2 now had a fixed frequency of $1000 \mathrm{~Hz}$; thus, for a given subject and number of cycles, S1 had only two possible frequencies, with a geometric mean of $1000 \mathrm{~Hz}$ and a distance corresponding to $2 \cdot \Delta F_{\text {rel }}$. Second, there was no 4-sec ISI; instead, we used a 100-msec ISI, which was not used in Experiment 1.

Among the 4 listeners who served as subjects, 2 (G.M. and L.D.) had been tested in Experiment 1. The other 2 listeners (E.D. and

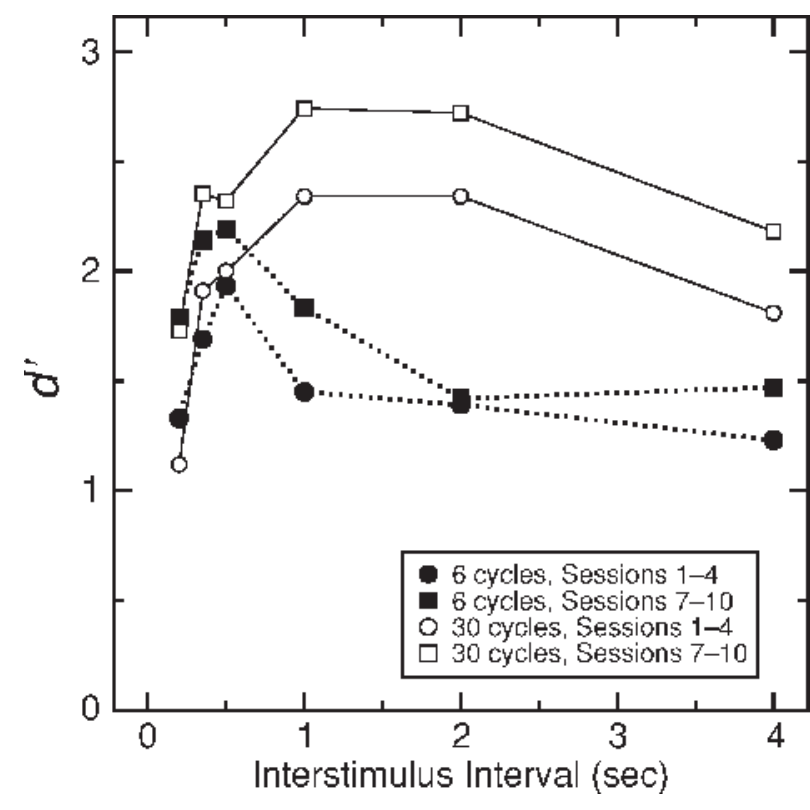

Figure 3. Effect of subjects' practice on $d^{\prime}$ in Experiment 1. The results obtained in Sessions 1-4 and 7-10 are plotted separately. Each data point represents the mean of eight $d^{\prime}$ values $(4 \mathrm{sub}$ jects $\times 2$ measurements of $d^{\prime}$ per subject). 


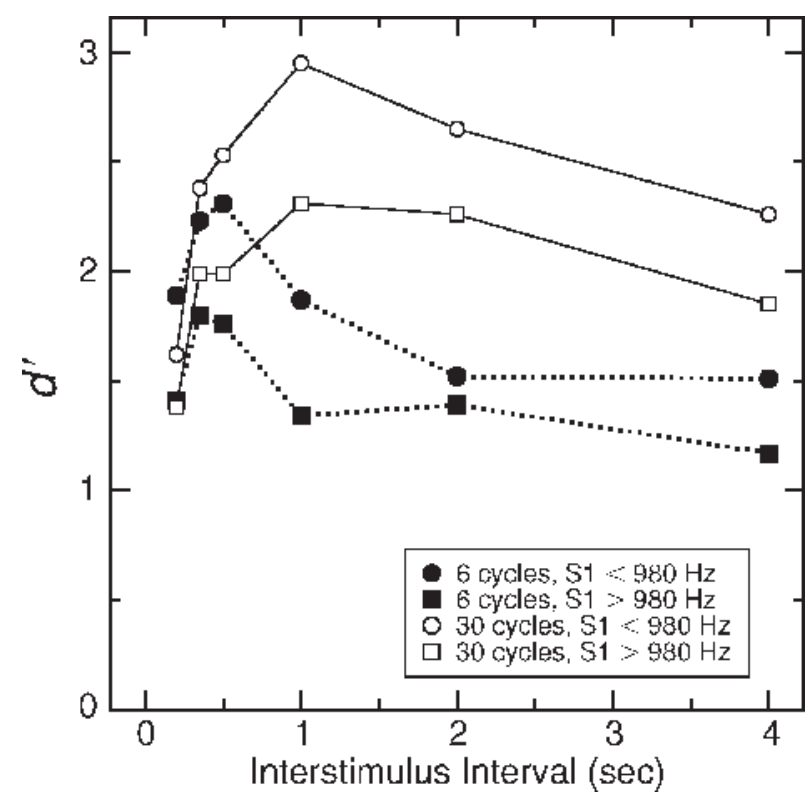

Figure 4. Dependence of $d^{\prime}$ on the frequency register of S1 (below $980 \mathrm{~Hz}$ vs. above $980 \mathrm{~Hz}$ ) in Experiment 1. Each data point represents the mean of four $d^{\prime}$ values (one value per subject).

N.P.) had no previous experience in psychophysical tasks; they were students without any known hearing deficits.

For each subject, the experiment proper again consisted of 9,000 trials $(2$ numbers of cycles $\times 6$ ISIs $\times 10$ blocks of 75 trials $)$ run in 10 sessions. These 10 sessions were again preceded by a preliminary stage intended to train the subjects and to select $\Delta F_{\text {rel }}$ values yielding a $d^{\prime}$ of about 2.0 for a $350-\mathrm{msec}$ ISI in both the 6-cycles condition and the 30 -cycles condition. The selected $\Delta F_{\text {rel }}$ values are indicated in Table 1.

\section{Results and Discussion}

The collected data were analyzed exactly as those of Experiment 1, and the results are displayed in Figure 5. As was expected, for the $350-\mathrm{msec}$ ISI the average value of $d^{\prime}$ was about the same in the two stimulus conditions and close to 2.0. As in Experiment 1, $d^{\prime}$ was a nonmonotonic function of the ISI for each number of cycles: When the ISI increased from $100 \mathrm{msec}$ to $2 \mathrm{sec}, d^{\prime}$ initially increased and then decreased. However, in striking contrast to our previous results, there was no significant interaction between ISI and the number of cycles $[F(5,20)<1]$. The optimal ISI did not appear to differ in the two stimulus conditions and could be assessed in both cases at about $400 \mathrm{msec}$. For the 6-cycles condition, this value was similar to that found in Experiment 1. For the 30-cycles condition, however, the optimal ISI appeared to be shorter in the present experiment than in Experiment 1.

The implications of this main finding will be considered later. Meanwhile, let us note that the noneffect of the stimulus condition on the optimal ISI confirms that an explanation of the previous effect in terms of forward masking would be inappropriate: The potential influence of a relatively peripheral phenomenon such as forward masking on the optimal ISI was the same in the two experiments.

In the present experiment, it was not obvious a priori that $d^{\prime}$ would decrease beyond some ISI. Instead, a plateau might have been obtained. Since the difference between the two possible S1 stimuli was twice as large as the difference between either of them and S2, the subjects might have performed the task by making absolute judgments on S1 rather than comparisons between S1 and S2. The fact that $d^{\prime}$ decreased beyond some ISI shows that this was not the case, presumably because the representations of the two possible S1 stimuli in the subjects" "long-term" memory were not accurate enough (see, in this respect, Hawkins \& Presson, 1977; Kallman \& Massaro, 1979).

Nevertheless, frequency discrimination performance was better here than in Experiment 1, since the values of $\Delta F_{\text {rel }}$ were about two times smaller (see Table 1) while the maximum values of $d^{\prime}$ were roughly similar (cf. Figures 2 and 5). This improvement is probably due mainly to the elimination of the roving procedure. If so, it was predictable from previous research (Berliner \& Durlach, 1973; Berliner et al., 1977; Harris, 1952; Oxenham \& Buus, 2000; Watson, Kelly, \& Wroton, 1976). The deleterious effect of roving on discrimination is liable to originate from various central factors. Oxenham and Buus conceptualized this effect as the addition of a stimulus-independent internal noise to the stimulus-dependent sensation noise. In accordance with this model, they found in a study on intensity discrimination that the relative effect of roving on discrimination thresholds was smaller for very short stimuli (having a poor discrimination threshold even in the absence of roving) than for longer stimuli (which were better discriminated in the absence of roving). In the present study, however, the relative effect of roving on frequency discrimination did not appear to be systematically smaller in the 6-cycles condition than in the 30-cycles condition: For subjects G.M. and L.D., on average, this effect could be estimated as a decrease of sensitivity $\left(d^{\prime} / \Delta F_{\text {rel }}\right.$ at the optimal ISI) by a factor of 1.90 in the 6-cycles condition versus 1.77 in the 30 -cycles condition. Thus, our data do not lend support to the model proposed by Oxenham and Buus.

\section{EXPERIMENT 3}

In the 30-cycles condition of Experiment 2, the optimal ISI was shorter than that in the corresponding condition of Experiment 1. It seems reasonable to think that this was the case because S1 had a smaller level of perceptual uncertainty in Experiment 2. We attempted to confirm this hypothesis in Experiment 3.

\section{Method}

Four listeners were tested in two new variants of the 30-cycles condition. The high- $U$ (high-uncertainty) variant replicated what had been done in the 30-cycles condition of Experiment 1, except that the ISI values were now 75, 150, 300, 600, 1,200, and $2,400 \mathrm{msec}$. In the low- $U$ (low-uncertainty) variant, the ISIs were the same as in the high-U variant, and the same was true for $\Delta F_{\text {rel }}$ (which 


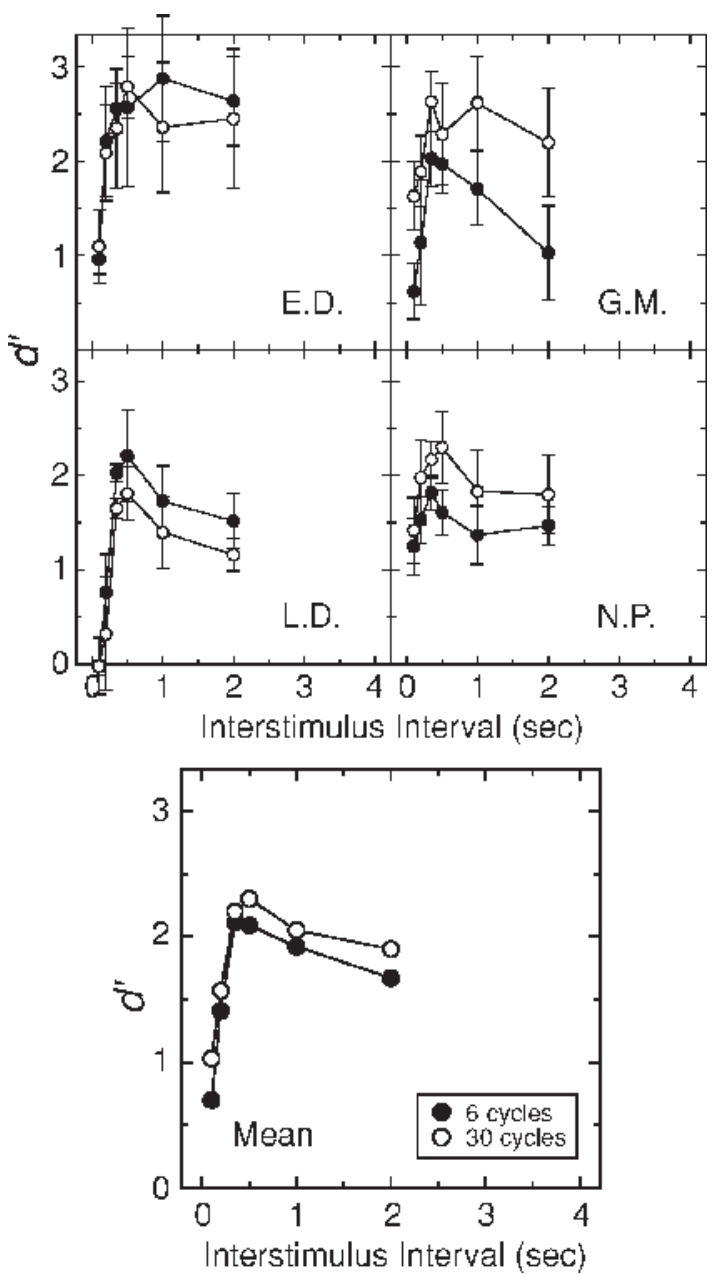

Figure 5. Results of Experiment 2: $d^{\prime}$ as a function of interstimulus interval in the 6-cycles condition (filled circles) and the 30cycles condition (open circles). The four upper panels display the individual data, which are averaged in the bottom panel. $d^{\prime}$ represents the relative discriminability of ascending and descending frequency shifts $(\mathbf{S} 2>S 1$ vs. S2 $<$ S1). The error bars have a total length of two standard deviations.

nonetheless varied across listeners). In addition, the frequency range of $\mathrm{S} 1$ was also the same in the two variants. However, whereas in the high- $\mathrm{U}$ variant $\mathrm{S} 1$ could take any possible frequency between 400 and $2400 \mathrm{~Hz}$, which made it highly uncertain, S2 could take only three possible frequencies in the low-U variant: 400, 2400, and $980 \mathrm{~Hz}$ (the geometric mean of 400 and $2400 \mathrm{~Hz}$ ). Moreover, in the low-U variant, the random choice of $\mathrm{S} 2$ on each trial was constrained by the exclusion of immediate repetitions; consequently, the objective uncertainty of S2 on a given trial amounted to only one bit, and that of S1 to two bits.

For each ISI and each listener, 750 low-U trials and 750 high-U trials were run in 10 sessions, consisting once more of alternating blocks of 75 trials. The 4 listeners serving as subjects were 3 students (G.L.S., C.F., and J.D.P.) with normal hearing and no previous experience in psychophysical tasks, and, again, author L.D. The experiment proper was preceded by a training phase of 3-6 sessions (depending on the listener). In the experiment proper, $\Delta F_{\text {rel }}$ was set to 12 cents for J.D.P., 13 cents for G.L.S., 14 cents for C.F., and 16 cents for L.D.

\section{Results and Discussion}

The results are displayed in Figure 6. Note first that performance was definitely better on low-U trials than on high-U trials. This highly significant effect $[F(1,4)=$ $318.4, p<10^{-4}$ ] is meaningful since $\Delta F_{\text {rel }}$ had the same values for the two types of trials. More importantly, there was also a significant interaction between uncertainty (low-U vs. high-U) and ISI $[F(5,20)=8.4, p<.001]$. The discrimination advantage resulting from a low degree of uncertainty was larger for short ISIs than for long ISIs. When uncertainty was high, the optimal ISI was, on average, close to $1 \mathrm{sec}$, as in Experiment 1. When uncertainty was low, the optimal ISI was shorter on average, as in Experiment 2. Overall, these data support the view that, in Experiments 1 and 2, the uncertainty of S1 had, per se, an influence on the optimal ISI as well as on the overall level of performance. On the other hand, it should be pointed out that the interaction between the factors of uncertainty and ISI observed in the present experiment is less spectacular than the interaction found in Experiment 1.

\section{GENERAL DISCUSSION}

In Experiment 1, we found that pitch discrimination was optimal for a shorter ISI when the stimuli consisted of 6 sine cycles than when they consisted of 30 sine cycles. Two hypotheses were put forth to account for this effect. First, it could be supposed that the crucial factor involved was the number of cycles itself - that is, the amount of temporal information on frequency provided to the pitch processor. According to the alternative hypothesis, the crucial factor was instead the perceptual uncertainty of $\mathrm{S} 1$, which could be considered as higher in the 30-cycles condition than in the 6-cycles condition even though the range of roving was physically the same in the two conditions. In the 30-cycles condition, S1 had a very high level of perceptual uncertainty insofar as, for the average subject, the roving range encompassed about 182 frequency difference limens $\left(d^{\prime}=2\right)$ at the optimal ISI. In the 6-cycles condition, the number of difference limens encompassed by the roving range was still large but considerably smaller (about 32).

In Experiment 2, the stimuli again consisted of either 6 or 30 sinusoidal cycles, but this time the perceptual uncertainty of S1 was minimal and independent of the number of cycles. The results showed that the optimal ISI was the same in the two stimulus conditions, thus suggesting that the crucial factor involved in Experiment 1 was the perceptual uncertainty of S1. This hypothesis was supported, to some extent, by Experiment 3. In the latter experiment, on the other hand, the effect of uncertainty on the optimal ISI was not extremely strong. Therefore, uncertainty may not have been the only source or even the main source of the strong effect observed in Experiment 1. If uncertainty was not the only source of the effect, any other factors involved remain to be identified.

A manipulation of stimulus uncertainty affects the predictability of a stimulus, but it can also affect its familiarity. These are two distinct variables. We surmise that the 

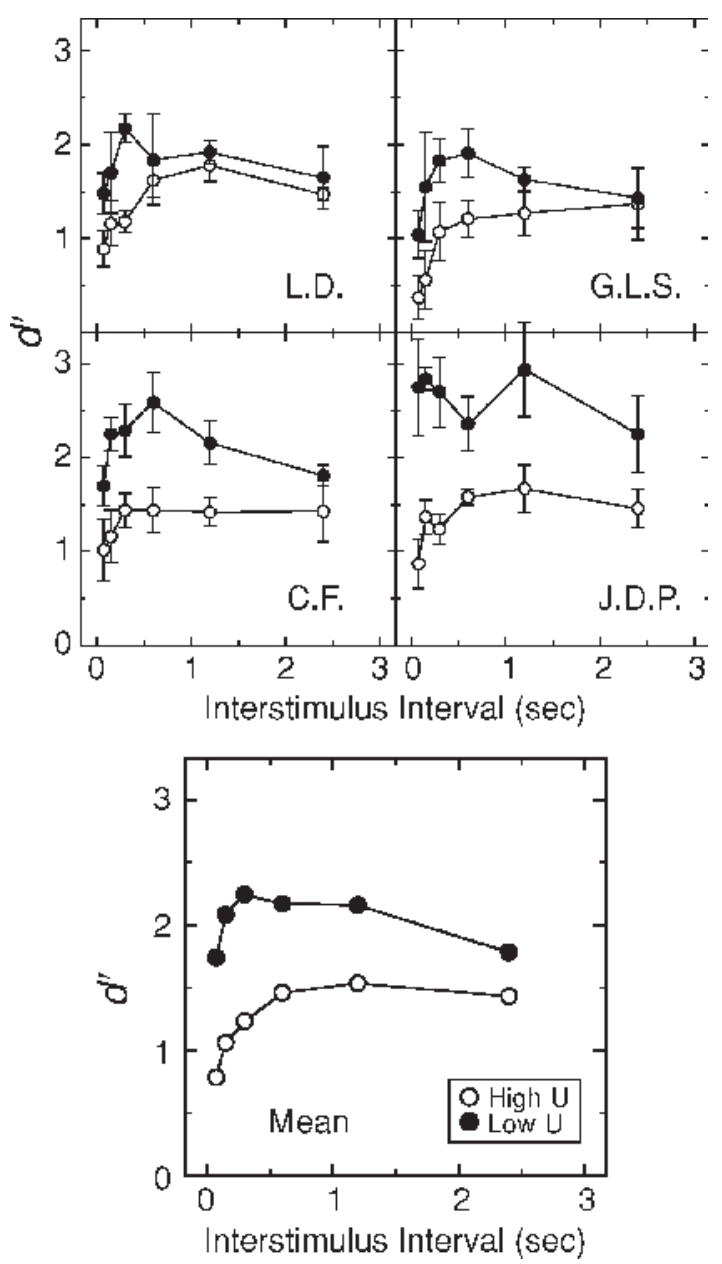

Figure 6. Results of Experiment 3: $d^{\prime}$ as a function of interstimulus interval in the high-U and low-U variants of the 30-cycles condition. The error bars have a total length of two standard deviations.

crucial one with regard to the optimal ISI is predictability, because, in Experiment 1, the optimal ISIs did not change as a function of practice even though the stimuli must have become more and more familiar and an overall increase of $d^{\prime}$ was indeed observed during the experiment (Figure 3). Likewise, in Experiment 3 there was no clear effect of practice on the optimal ISIs. It would be interesting to dissociate the predictability factor from the familiarity factor. Predictability can be manipulated independently of familiarity by selecting S1 from a constant set of possible stimuli and presenting before it a pitch cue with a variable degree of validity (see, in this respect, Demany, Montandon, \& Semal, 2004; Hafter, Schlauch, \& Tang, 1993; Mondor \& Bregman, 1994).

The present study was intended to test a theory according to which the duration required to perceive a short sound as accurately as possible is a constant corresponding to the fixed temporal span of a PPAS. Clearly, our results are at odds with this theory: They show that an optimal perception of pitch does not require an invariable amount of time. On the other hand, our results, like previous results, do support the idea that human listeners possess a PPAS. If such a store did not exist, the discriminability of two short sounds could not possibly increase when the ISI increases, in the absence of a forward masking effect. What our results seem to call for is a reconsideration of the PPAS properties.

Exactly what does happen to an auditory trace in the PPAS? A simple hypothesis, implicitly adopted by Massaro (1972) and Massaro and Loftus (1996), is that the trace does not change and provides invariable information as long as it can be retained in this store. Under this hypothesis, one may suppose at first that the retention delay is a constant and does not depend on the stimulus. However, the corresponding option is incompatible with our results, since it implies that in Experiment $1 d^{\prime}$ should have started to decline beyond a constant ISI (the same ISI for both stimulus conditions). An alternative option is that the retention delay actually depends on the stimulus. A priori, this idea makes sense: If what occurs in the PPAS is a persistence of neural activity produced by the stimulus, the duration of the persistence in question may be stimulus dependent just because the neural activity itself will be stimulus dependent. However, this alternative option is again hard to reconcile with our results, since the ISI beyond which $d^{\prime}$ started to decline was stimulus dependent in our first experiment but not in the second experiment, although there was a similar difference between the two stimulus conditions of each experiment. In conclusion, therefore, it seems basically wrong to hypothesize that an auditory trace provides invariable information as long as it stays within the PPAS.

Let us suppose instead that the PPAS is an imperfect memory store in which a trace becomes more and more inaccurate throughout its existence. Let us suppose also, as Massaro (1972) did, that the perceptual processing of a short stimulus is a temporally extended readout of the information provided by its trace in the PPAS. It is then possible to account for our results, at least qualitatively. In a discrimination experiment such as those reported here, the optimal ISI will correspond to an optimal compromise between two conflicting requirements: on the one hand, the need for time for the readout process, and on the other hand the need to process a trace while it is as fresh (and therefore as accurate) as possible. If the rate at which a trace decays in the PPAS does not depend on the stimulus, but the speed of the readout process is a function of the effective uncertainty of the stimulus, then the level of this effective uncertainty may well have an influence on the optimal ISI: A very high uncertainty may slow down the readout process and thus increase the optimal ISI.

Admittedly, one problem with this interpretation is that the nature of the readout process is unclear. In spite of Attneave's (1961) plea "in defense of homunculi," the human brain is probably not occupied by a little man watching a cortical screen on which a short tone would leave an unstable trace. Therefore, if the very notion of a readout process is indeed warranted, one should endeavor to make of it more than an anthropomorphic metaphor. 


\section{REFERENCES}

Attneave, F. (1961). In defense of homunculi. In W. A. Rosenblith (Ed.), Sensory communication contributions (pp. 777-782). Cambridge, MA: MIT Press.

Berliner, J. E., \& Durlach, N. I. (1973). Intensity perception: IV. Resolution in roving-level discrimination. Journal of the Acoustical Society of America, 53, 1270-1287.

Berliner, J. E., Durlach, N. I., \& Braida, L. D. (1977). Intensity perception: VII. Further data on roving-level discrimination and the resolution and bias edge effects. Journal of the Acoustical Society of America, 61, 1577-1585.

CARbotte, R. M. (1973). Retention of time information in forced-choice duration discrimination. Perception \& Psychophysics, 14, 440-444.

Cowan, N., Saults, J. S., \& Nugent, L. D. (1997). The role of absolute and relative amounts of time in forgetting within immediate memory: The case of tone-pitch comparisons. Psychonomic Bulletin \& Review, 4, 393-397.

Demany, L., Montandon, G., \& Semal, C. (2004). Pitch perception and retention: Two cumulative benefits of selective attention. Perception \& Psychophysics, 66, 609-617.

Demany, L., Montandon, G., \& Semal, C. (2005). Internal noise and memory for pitch. In D. Pressnitzer, A. de Cheveigné, S. McAdams, \& L. Collet (Eds.), Auditory signal processing: Physiology, psychoacoustics, and models (pp. 230-236). New York: Springer.

Goldstein, J. L., \& SRulovicz, P. (1977). Auditory-nerve spike intervals as an adequate basis for aural frequency measurement. In E. F. Evans \& J. P. Wilson (Eds.), Psychophysics and physiology of hearing (pp. 337-346). London: Academic Press.

Hafter, E. R., Schlauch, R. S., \& TANG, J. (1993). Attending to auditory filters that were not stimulated directly. Journal of the Acoustical Society of America, 94, 743-747.

HARRIS, J. D. (1952). The decline of pitch discrimination with time. Journal of Experimental Psychology, 43, 96-99.

Hawkins, H. L., \& Presson, J. C. (1977). Masking and preperceptual selectivity in auditory recognition. In S. Dornic (Ed.), Attention and performance VI (pp. 195-211). Hillsdale, NJ: Erlbaum.

Kallman, H. J., \& Massaro, D. W. (1979). Similarity effects in backward recognition masking. Journal of Experimental Psychology: Human Perception \& Performance, 5, 110-128.

Massaro, D. W. (1970). Preperceptual auditory images. Journal of Experimental Psychology, 85, 411-417.

Massaro, D. W. (1972). Stimulus information vs processing time in auditory pattern recognition. Perception \& Psychophysics, 12, 50-56.

Massaro, D. W. (1975). Backward recognition masking. Journal of the Acoustical Society of America, 58, 1059-1065.

Massaro, D. W., \& IDSON, W. L. (1977). Backward recognition masking in relative pitch judgments. Perceptual \& Motor Skills, 45, 87-97.

Massaro, D. W., \& Loftus, G. R. (1996). Sensory and perceptual storage. In E. L. Bjork \& R. A. Bjork (Eds.), Memory (pp. 67-99). San Diego: Academic Press.

Mondor, T. A., \& Bregman, A. S. (1994). Allocating attention to frequency regions. Perception \& Psychophysics, 56, 268-276.

Moore, B. C. J. (1973). Frequency difference limens for short-duration tones. Journal of the Acoustical Society of America, 54, 610-619.

Oxenham, A. J., \& BuUs, S. (2000). Level discrimination of sinusoids as a function of duration and level for fixed-level, roving-level, and across-frequency conditions. Journal of the Acoustical Society of America, 107, 1605-1614.

Pisoni, D. B. (1973). Auditory and phonetic memory codes in the discrimination of consonants and vowels. Perception \& Psychophysics, 13, 253-260.

Ronken, D. A. (1972). Changes in frequency discrimination caused by leading and trailing tones. Journal of the Acoustical Society of America, 51, 1947-1950.

Scharf, B. (1978). Loudness. In E. C. Carterette \& M. P. Friedman (Eds.), Hearing (pp. 187-242). New York: Academic Press.

Small, A. M., \& Campbell, R. A. (1962). Temporal differential sensitivity for auditory stimuli. American Journal of Psychology, 75 401-410.

SoRKIN, R. D. (1966). Temporal interference effects in auditory amplitude discrimination. Perception \& Psychophysics, 1, 55-58.

TANner, W. P. (1961). Physiological implications of psychophysical data. Annals of the New York Academy of Sciences, 89, 752-765.

TAYLOR, M. M., \& SMITH, S. M. (1975). Monaural detection with contralateral cue: V. Interstimulus interval in MDCC and amplitude discrimination. Journal of the Acoustical Society of America, 57, 15001511.

Turner, C. W., Zeng, F. G., Relkin, E. M., \& Horwitz, A. R. (1992). Frequency discrimination in forward and backward masking. Journal of the Acoustical Society of America, 92, 3102-3108.

Watson, C. S., Kelly, W. J., \& Wroton, H. W. (1976). Factors in the discrimination of tonal patterns: II. Selective attention and learning under various levels of stimulus uncertainty. Journal of the Acoustical Society of America, 60, 1176-1186.

\section{NOTES}

1. The relation between $d^{\prime}$ and $P(\mathrm{C})$ is of course strongly nonlinear at high performance levels.

2. Data from Experiment 1 were presented at the 13 th International Symposium on Hearing (Dourdan, France, August 2003) and published in the proceedings of that meeting (Demany, Montandon, \& Semal, 2005). In contrast to the present article, the previous paper focused on the decay of frequency discrimination performance at long ISIs.

3. Small and Campbell (1962) measured the duration difference limens of short tone bursts with a rectangular amplitude envelope and a frequency of 250 or $5000 \mathrm{~Hz}$ (using stimuli equated in power, rather than in energy, within trials). They found that the Weber fraction corresponding to the duration difference limen was about 0.3 for a standard duration of $4 \mathrm{msec}$ (close to the average stimulus duration in our 6-cycles condition) and about 0.1 for a standard duration of $40 \mathrm{msec}$ (close to the average stimulus duration in our 30 -cycles condition). These two Weber fractions are, respectively, 5.2 and 7.8 times larger than those corresponding to the average relative frequency shifts produced in Experiment 1 . Note that the detectability of differences in duration is probably better for stimuli with a rectangular envelope than for stimuli with the smoother envelope used in our experiments.

(Manuscript received April 2, 2004 revision accepted for publication February 16, 2005.) 